\title{
Three-Dimensional Second-Order Accurate Difference Schemes for Discontinuous Hydrodynamic Flows*
}

\author{
By Ephraim L. Rubin and Stanley Preiser
}

\begin{abstract}
In this paper, we show how a second-order accurate two-step method used in the numerical computation of hydrodynamic flows may be derived directly from the integral conservation laws. A necessary and sufficient condition for stability for the linearized equations is derived for the three-dimensional Cartesian coordinate case.
\end{abstract}

I. Introduction. In 1960 Lax and Wendroff [2] presented a second-order accurate scheme for the numerical computation of hydrodynamic flows (neglecting various stresses and heat conduction). An important feature of the method was that the differential equations were written as a first-order system in conservation form. The difference scheme was derived by expanding the solution in a Taylor series in the time variable up to terms of second order. The method involved the computation of matrices, the determinants of which were the Jacobians of certain transformations.

Richtmyer [3] presented a two-step method, for problems in two-space dimensions, explicit like the Lax-Wendroff, but which required no matrix calculations and had the same order of accuracy. A third explicit two-step method, avoiding matrix calculations, was used by Rubin and Burstein [4] and in the latter paper all three schemes were compared. In Section II of this paper, we shall show how the twostep Lax-Wendroff method as given by Richtmyer can be generalized to threespace dimensions and time. The result follows, with suitable approximations, directly from the integral conservation laws. The motivation for this approach is twofold:

(1) The conservation laws are, in fact, integral in nature.**

(2) Alternative quadrature methods immediately suggest themselves. In particular, we have used the midpoint and rectangular rules for numerical integration. This approach, however, may be used to derive approximations of higher order than two. It is to be noted that the analysis given here depends on the differentiability of the functions. The applications of interest, however, are to discontinuous flows. The justification for the use of this approximation is given in [2] where it is proved that if a solution to the finite-difference equations exists, then the LaxWendroff scheme converges to a weak solution of the conservation laws. In [1], Anderson, Preiser, and Rubin showed how the hydrodynamic equations could be written in conservation form for arbitrary orthogonal curvilinear coordinate sys-

Received August 16, 1968, revised April 24, 1969.

AMS Subject Classifications. Primary 6567, 7645, 7635, 7665.

Key Words and Phrases. Fluid dynamics, stability, conservation laws.

* This research was conducted under the sponsorship of the Office of Naval Research under Contract No. Nonr 839(34), Project No. NR 061-135.

** Truesdell and Toupin [5, p. 232] attribute the view that all natural laws should be expressed as integrals to the Göttingen lectures of Hilbert. 
tems. The derivation of Section II is then immediately applicable to any such coordinate system. The application of the midpoint rule to one- and two-space dimensions for Lagrangian coordinates appeared in a report by Taub [6].

In Section III we derive a necessary condition for stability for the linearized equations in three-dimensional Cartesian coordinates. A theorem of Kreiss [8] proves sufficiency and the Lax-Richtmyer theorem guarantees convergence, for this linear case.

II. Conservation Laws, Difference Schemes and Stability. The hydrodynamic conservation equations, in three dimensions, for perfect fluids in the absence of external and dissipative forces may be written in the following way:

$$
\begin{aligned}
& \int_{v(t)}\left\{\frac{\partial \rho}{\partial t}+\operatorname{div}(\rho u)\right\} d v=0, \\
& \int_{v(t)}\left\{\frac{\partial(\rho u)}{\partial t}+\operatorname{div}(\rho u u+p I)\right\} d v=0, \\
& \int_{v(t)}\left\{\frac{\partial E}{\partial t}+\operatorname{div}(u[E+p])\right\} d v=0,
\end{aligned}
$$

$\rho, u, E$, and $p$ are the density, velocity vector, total energy per unit volume and pressure respectively. $I$ is the identity tensor, $u u$ is a dyadic product*** and $v(t)$ is the time dependent material volume. We assume that $p$ may be eliminated by means of an independent equation of state relating $p$ and the other variables. The above system of five equations in Cartesian coordinates may be written as follows: $\dagger$

$$
\int_{v(t)}\left\{w_{, t}+f_{, x^{r}}^{r}\right\} d v=0
$$

$w$ is a 5 component vector function of $x$ and $t,\left(\rho, \rho u^{1}, \rho u^{2}, \rho u^{3}, E\right)$ and $f$ is a nonlinear function of $w$. For continuous integrands, the integral equations are equivalent to the following differential equations

$$
w_{, t}+f_{, x^{r}}^{r}=0 \text {. }
$$

Across a surface of discontinuity, the Rankine-Hugoniot relations, which are embedded in the integral formulation, must be appended to the differential equations.

Lax and Wendroff consider the system of equations (2.3). They assume that $w(x, t)$ is an exact smooth solution of that system and expanded it in a Taylor series up to terms of second order. In one dimension

$$
w(x, t+\Delta t)=w(x, t)+\Delta t w_{, t}+\frac{1}{2}(\Delta t)^{2} w_{, t t}+O\left(\Delta^{3}\right) .
$$

Using (3) they express the time-derivatives as space derivatives:

$$
\begin{aligned}
w_{, t} & =-f_{, x} \\
w_{, t t} & =-f_{, x t}=-f_{, t x}=\left(A f_{, x}\right)_{, x} .
\end{aligned}
$$

*** If $u$ is a column vector with components $\left(u^{1}, u^{2}, u^{3}\right)$, then $u u$ represents the matrix product $u u^{T}$ where $u^{T}$ is the transpose of $u$.

$\dagger$ The Latin indices take on the values 1, 2, 3. The Einstein summation convention and the comma notation for differentiation are employed, thus, $f_{, x^{r}}^{r}=\partial f^{1} / \partial x^{1}+\partial f^{2} / \partial x^{2}+\partial f^{3} / \partial x^{3}$. 
$A$ is the matrix whose determinant is the Jacobian of $f$ with respect to $w$. Hence:

$$
w(x, t+\Delta t)=w(x, t)-\Delta t f_{, x}+\frac{1}{2}(\Delta t)^{2}\left(A f_{, x}\right)_{, x}+O\left(\Delta t^{3}\right) .
$$

The finite-difference analogue of this equation is found by approximating $f_{, x}$ and $\left(A f_{, x}\right)_{, x}$.

In [3] Richtmyer presented his two-step version of the Lax-Wendroff scheme for one- and two-space dimensions. In one dimension it may be written

$$
\begin{aligned}
\bar{w}(t+\Delta t, x)= & \frac{1}{2}(w(t, x+\Delta x)+w(t, x-\Delta x)) \\
& -\frac{\Delta t}{2 \Delta x}[f(w(t, x+\Delta x))-f(w(t, x-\Delta x))] \\
w(t+2 \Delta t, x)= & w(t, x)-\frac{\Delta t}{\Delta x}[f(\bar{w}(t+\Delta t, x+\Delta x))] \\
& -f(\bar{w}(t+\Delta t, x-\Delta x))] .
\end{aligned}
$$

The bars signify intermediate values. These intermediate values are calculated using Eq. (2.4a) which is a first-order difference approximation. Equation (2.4b) is second-order accurate.

Consider now the following three integrals which express the hydrodynamic conservation laws in an arbitrary orthogonal coordinate system.

$$
\int_{v(t)}\left\{\left((g)^{1 / 2} \rho\right)_{, t}+\left((g)^{1 / 2} u^{r}\right)_{, r}\right\} d x^{1} d x^{2} d x^{3}=0,
$$

$$
\begin{gathered}
\int_{r(t)}\left\{\left((g)^{1 / 2} \xi_{r} \rho u^{r}\right)_{, t}+\left((g)^{1 / 2} \xi_{r}\left[\frac{\rho u^{r} u^{s}}{\sqrt{ } g_{r r} \sqrt{ } g_{s s}}+\frac{p \delta^{r s}}{\sqrt{ } g_{r r} \sqrt{ } g_{s s}}\right]\right)_{, s}\right\} d x^{1} d x^{2} d x^{3}=0, \\
\int_{v(t)}\left\{\left((g)^{1 / 2} E\right)_{, t}+\left((g)^{1 / 2} u^{r}[E+p]\right)_{, r}\right\} d x^{1} d x^{2} d x^{3}=0 .
\end{gathered}
$$

In these equations $g$ is the determinant of $g_{r s}$, the flat metric of Newtonian space with the coordinates $\left(x^{1}, x^{2}, x^{3}\right)$. The covector $\xi_{r}=g_{r s} \xi_{s}$ satisfies Eq. (2.6) which is known as Killing's equation [7]

$$
\xi_{r ; s}+\xi_{s ; r}=0 \text {. }
$$

The semicolon in (2.6) denotes covariant differentiation and $\delta$ is the Kronecker delta. $u^{r}$ and $u^{s}$ are the physical velocities and not the tensor components; the $d x^{i}$, however, are the tensor components of the volume element. In [1], explicit examples for cylindrical and spherical coordinates are given.

We write down the analogues of Eqs. (2.4a) and (2.4b) for the 5-component vector $w:\left((g)^{1 / 2} \rho,(g)^{1 / 2} \rho u^{r} \xi_{r},(g)^{1 / 2} E\right)$ appearing in the integrands of Eqs. (2.5a, b, c) and the corresponding function $f(w)$

$$
w(t+\Delta t, x)=\left(I+Q_{1}\right) w(t, x)-\Delta t S(t, x),
$$

where

$$
\begin{aligned}
\lambda_{j} & =\frac{\Delta t}{\Delta x^{j}}, \quad S(t, x)=\sum D_{0 j} f^{j}(w(t, x)), \\
Q_{1} & =\frac{1}{6} \sum\left(\Delta x^{j}\right)^{2} D_{+j} D_{-j} .
\end{aligned}
$$


Here $x$ represents the three-dimensional point $\left(x^{1}, x^{2}, x^{3}\right)$, the difference operators are defined by

$$
\begin{aligned}
2 \Delta x D_{0 j} v(x) & =v\left(x+\Delta x e_{j}\right)-v\left(x-\Delta x e_{j}\right), \\
\Delta x D_{+j} v(x) & =v\left(x+\Delta x e_{j}\right)-v(x), \\
\Delta x D_{-j} v(x) & =v(x)-v\left(x-\Delta x e_{j}\right),
\end{aligned}
$$

where $e_{j}$ is the unit vector in the $x^{j}$ direction.

$$
w(t+2 \Delta t, x)=w(t, x)-2 \Delta t S(t+\Delta t, x) .
$$

Equation (2.7b) may be derived by applying the midpoint quadrature rule to the time integral of the integral conservation laws (2.5a, b, c). Assuming the validity of the interchange of the order of integration one of the integrals may be evaluated exactly. For sufficiently smooth integrands application of the midpoint rule to the remaining integrals gives $(2.7 \mathrm{~b})$.

The manner in which the first term on the right-hand side of $(2.7 \mathrm{a})$ is evaluated allows for some arbitrariness. A direct application of the rectangular rule to the time integral of $(2.5 \mathrm{a}, \mathrm{b}, \mathrm{c})$ after evaluating one of the integrals leads to $(2.7 \mathrm{a})$ with $Q_{1}=0$ which is unconditionally unstable. Our choice is guided by stability requirements. Equation (2.7a) as written is conditionally stable.

\section{A Necessary and Sufficient Condition for Stability in Cartesian Coordinates.} For the stability discussion of the three-dimensional Cartesian case we linearize the equations, i.e., we write

$$
f_{j, x^{k}}^{i}=A_{l} w_{l, x^{k}}
$$

where $A_{l}=\left(\partial f_{j}{ }^{i} / \partial w_{l}\right)$ are the matrices whose determinants are the Jacobians of the transformation and are regarded as locally constant.

To calculate the amplification matrix we try a solution of the form

$$
w(t, x, y, z)=w_{0}{ }^{t} \exp \left[i a_{1} \Delta x^{1}+i a_{2} \Delta x^{2}+i a_{3} \Delta x^{3}\right]
$$

and substitute into Eq. (2.7b) using Eqs. (2.7a) and (3.1). We shall consider the case where all the $\lambda_{i}$ 's are equal. The amplification matrix, $G$, is then given by the following expression:

$$
\begin{aligned}
G= & I-i\left[\cos \xi_{1}+\cos \xi_{2}+\cos \xi_{3}\right]\left[\frac{2}{3} \frac{\Delta t}{\Delta x}\left(A_{1} \sin \xi_{1}+A_{2} \sin \xi_{2}+A_{3} \sin \xi_{3}\right)\right] \\
& -2\left[\frac{\Delta t}{\Delta x}\left(A_{1} \sin \xi_{1}+A_{2} \sin \xi_{2}+A_{3} \sin \xi_{3}\right)\right]^{2} .
\end{aligned}
$$

Here

$$
\xi_{1}=a_{1} \Delta x^{1}, \xi_{2}=a_{2} \Delta x^{2}, \xi_{3}=a_{3} \Delta x^{3} .
$$

The amplification matrix may also be derived in the following way which will be useful for the sufficiency part of the stability proof. $\dagger \dagger$ To linearize $(2.7 \mathrm{a}, \mathrm{b})$ we replace $S(t)$ by $Q=\sum A_{j} D_{0 j}$. Combining (2.7a) and (2.7b) leads to

$\dagger \dagger$ We are grateful to the reviewer for pointing this out to us. 


$$
w(t+2 \Delta t, x)=\left[I-2 \Delta t Q\left(I+Q_{1}\right)+2(\Delta t)^{2} Q^{2}\right] w(t, x) .
$$

Noting that the Fourier transforms of $Q$ and $Q_{1}$ are

$$
\hat{Q}=i \sum \frac{A_{j} \sin \xi_{j}}{\Delta x^{j}}, \quad \hat{Q}_{1}=-\frac{2}{3} \sum \sin ^{2} \frac{\xi_{j}}{2}=-I+\frac{1}{3} \sum \cos \xi_{j}
$$

we get (3.2) directly, for the case where the $\Delta x^{j}$ are all equal.

Rather than calculate the eigenvalues of $G$ we shall consider the eigenvalues of another matrix $G^{\prime}$ obtained by writing the equations in terms of density, velocity and pressure. This leads to new matrices $A_{1}{ }^{\prime}, A_{2}{ }^{\prime}$, and $A_{3}{ }^{\prime}$. They are related to $A_{1}, A_{2}$, and $A_{3}$ by a similarity transformation so that the stability discussion is unaffected by this transformation. In matrix form, for Cartesian coordinates,

$$
\begin{gathered}
\frac{\partial w^{\prime}}{\partial t}+A_{1}{ }^{\prime} \frac{\partial w^{\prime}}{\partial x}+A_{2} \frac{\partial w^{\prime}}{\partial y}+A_{3}{ }^{\prime} \frac{\partial w^{\prime}}{\partial z}=0 \\
w^{\prime}=\left[\begin{array}{c}
\rho \\
u \\
v \\
w \\
E
\end{array}\right], \quad A_{1}{ }^{\prime}=A_{1}{ }^{\prime}\left(w^{\prime}\right)=\left[\begin{array}{lllll}
u & \rho & 0 & 0 & 0 \\
0 & u & 0 & 0 & \frac{1}{\rho} \\
0 & 0 & u & 0 & 0 \\
0 & 0 & 0 & u & 0 \\
0 & \rho c^{2} & 0 & 0 & u
\end{array}\right], \\
A_{2}{ }^{\prime}=A_{2}{ }^{\prime}\left(w^{\prime}\right)=\left[\begin{array}{lllll}
v & 0 & \rho & 0 & 0 \\
0 & v & 0 & 0 & 0 \\
0 & 0 & v & 0 & \frac{1}{\rho} \\
0 & 0 & 0 & v & 0 \\
0 & 0 & \rho c^{2} & 0 & v
\end{array}\right], \\
A_{3}{ }^{\prime}=A_{3}{ }^{\prime}\left(w^{\prime}\right)=\left[\begin{array}{lllll}
w & 0 & 0 & \rho & 0 \\
0 & w & 0 & 0 & 0 \\
0 & 0 & w & 0 & 0 \\
0 & 0 & 0 & w & \frac{1}{\rho} \\
0 & 0 & 0 & \rho c^{2} & w
\end{array}\right] .
\end{gathered}
$$

Let

$$
M=A_{1}{ }^{\prime} \sin \xi_{1}+A_{2}{ }^{\prime} \sin \xi_{2}+A_{3}{ }^{\prime} \sin \xi_{3} .
$$

Consider a line with direction numbers $\sin \xi_{1}$, $\sin \xi_{2}$, and $\sin \xi_{3}$. Then

$$
\begin{aligned}
\cos r & =\frac{\sin \xi_{1}}{\left(\sin ^{2} \xi_{1}+\sin ^{2} \xi_{2}+\sin ^{2} \xi_{3}\right)^{1 / 2}}, \quad \cos s=\frac{\sin \xi_{2}}{\left(\sin ^{2} \xi_{1}+\sin ^{2} \xi_{2}+\sin ^{2} \xi_{3}\right)^{1 / 2}}, \\
\cos t & =\frac{\sin \xi_{3}}{\left(\sin ^{2} \xi_{1}+\sin ^{2} \xi_{2}+\sin ^{2} \xi_{3}\right)^{1 / 2}} ;
\end{aligned}
$$


$\cos r, \cos s$, and $\cos t$ are therefore the direction cosines of this line. From (3.4) $M=\left(\sin ^{2} \xi_{1}+\sin ^{2} \xi_{2}+\sin ^{2} \xi_{3}\right)^{1 / 2}\left[\begin{array}{lllll}u^{\prime} & \cos r & \cos s & \cos t & 0 \\ 0 & u^{\prime} & 0 & 0 & \frac{1}{\rho} \cos r \\ 0 & 0 & u^{\prime} & 0 & \frac{1}{\rho} \cos s \\ 0 & 0 & 0 & u^{\prime} & \frac{1}{\rho} \cos t \\ 0 & \rho c^{2} \cos r & \rho c^{2} \cos s & \rho c^{2} \cos t & u^{\prime}\end{array}\right]$,

where $u^{\prime}=u \cos r+v \cos s+w \cos t$ is the component of the velocity $\mathbf{V}$ in the above direction.

The eigenvalues of $M$ are

$$
\left(\sin ^{2} \xi_{1}+\sin ^{2} \xi_{2}+\sin ^{2} \xi_{3}\right)^{1 / 2}\left\{\begin{array}{l}
u^{\prime} \\
u^{\prime} \\
u^{\prime} \\
u^{\prime}+c \\
u^{\prime}-c
\end{array}\right\} .
$$

Let $\mu=(\Delta t / \Delta x) K$, where $K$ is an eigenvalue of $M$. The eigenvalues of $G$ are

$$
\begin{aligned}
g & ={ }_{1}-i \frac{2}{3} \mu\left(\cos \xi_{1}+\cos \xi_{2}+\cos \xi_{3}\right)-2 \mu^{2}, \\
|g|^{2} & =\left(1-2 \mu^{2}\right)^{2}+\frac{4}{9} \mu^{2}\left(\cos \xi_{1}+\cos \xi_{2}+\cos \xi_{3}\right)^{2} \\
& \leqq\left(1-2 \mu^{2}\right)^{2}+\frac{12}{9} \mu^{2}\left(\cos ^{2} \xi_{1}+\cos ^{2} \xi_{2}+\cos ^{2} \xi_{3}\right) \\
& =1-\frac{12}{9} \mu^{2}\left[\left(\sin ^{2} \xi_{1}+\sin ^{2} \xi_{2}+\sin ^{2} \xi_{3}\right)-3 \mu^{2}\right] \\
& \left.=1-\frac{4}{3} \mu^{2}\left[\sin ^{2} \xi_{1}+\sin ^{2} \xi_{2}+\sin ^{2} \xi_{3}\right]\left[\begin{array}{l}
u^{\prime} \\
u^{\prime} \\
u^{\prime} \\
u^{\prime}+c \\
u^{\prime}-c
\end{array}\right\}\right] .
\end{aligned}
$$

The largest values of the quantities in the curly bracket is $|\mathbf{V}|+c$. Hence $|g|^{2}$ $\leqq 1$ if $(\Delta t / \Delta x)(|\mathbf{V}|+c)<1 / \sqrt{ } 3$.

For Cartesian coordinates the stability condition is $(\Delta t / \Delta x)(|v|+c)<1 / \sqrt{ } n$ where $n=1,2,3$ is the dimension of the space. 
To prove sufficiency, we use a theorem of Kreiss [8]. $\dagger \dagger \dagger$ Consider the following hyperbolic system of equations with constant coefficients $w_{, t}=P(\partial / \partial x) w$ and the difference approximation to it

$$
v(t+\Delta t, x)=[I+Q(\Delta x D)] v(t, x) .
$$

$Q(\Delta x, D)$ is a polynomial in $\Delta x D_{0 j}, \Delta x D_{+j}$ and $\Delta x D_{-j}$ with constant matrix coefficients.

The difference approximation (3.5) is called dissipative of order $2 r, r$ a nonnegative integer, if there exists a constant $\delta<0$ such that for all $\xi$ with $|\xi| \leqq \pi$

$$
\left|\mathcal{F C}_{j}(\xi)\right| \leqq 1-\delta|\xi|^{2 r}
$$

where $\mathfrak{H}_{j}=\mathfrak{F}_{j}(I+\hat{Q})$ is an eigenvalue of $I+\hat{Q}$ and $I+\hat{Q}$ is the Fourier transform of $I+Q(\Delta x D) . \xi=\omega \Delta x$, where $\omega$ is the dual variable.

Theorem 5 of Kreiss states: Let the difference approximation (3.5) be dissipative of order $2 r$ and assume that for $|\xi| \leqq \pi, I+\hat{Q}$ can be written in the form

$$
I+\hat{Q}=I+\sum_{\nu=1}^{2 r-2} \frac{1}{\nu !}(\lambda \hat{P}(\xi))^{\nu}+\hat{Q}_{2 r}(\xi),\left|\hat{Q}_{2 r}\right|=O\left(|\xi|^{2 r}\right)
$$

Then it is stable.

We now observe that (3.3) may be written

$$
w(t+2 \Delta t, x)=\left[I-2 \Delta t Q\left(I+Q_{1}\right)+2(\Delta t)^{2}\left(\left(Q\left(I+Q_{1}\right)\right)^{2}-Q_{2}\right)\right] w(t, x)
$$

with

$$
Q_{2 r}=2(\Delta t)^{2}\left(2 Q^{2} Q_{1}+Q^{2} Q_{1}^{2}\right)
$$

Since

$$
\left|\hat{Q}_{2 r}\right| \leqq \text { constant }|\xi|^{4}
$$

we have satisfied the conditions of Theorem 5 which proves that the linearized approximation is stable.

Polytechnic Institute of Brooklyn

Brooklyn, New York 11201

1. J. L. Anderson, S. Preiser \& E. L. Rubin, "Conservation form of the equations of hydrodynamics in curvilinear coordinate systems," J. Computational Phys., v. 2, 1968, pp. 279-287.

2. P. D. Lax \& B. Wendroff, "Systems of conservation laws," Comm. Pure Appl. Math., v. 13, 1960, pp. 217-237. MR 22 \#11523.

3. R. D. Richtm Yer, A Survey of Difference Methods for Non-Steady Fluid Dynamics, NCAR Technical Note $63-2,1962$.

4. F. L. RUBin \& S. Z. Burstein, "Difference methods for the inviscid and viscous equations of a compressible gas," J. Computational Phys., v. 2, 1967, pp. 178-196.

5. C. TRuespell \& R. Toupin, The Classical Field Theories, Handbuch der Physik, Band III/I, Springer-Verlag, Berlin, 1960. MR 22 \#8778.

6. A. H. TAUB, On the Derivation of Difference Equations for Hydrodynamics, Los Alamos Scientific Laboratory Report LA-2073, 1957.

7. L. P. Ersenhart, Continuous Groups of Transformations, Dover, New York, 1961, p. 208. MR 23 \#A1328.

8. H.-O. KREISs, "On difference approximations of the dissipative type for hyperbolic differential equations," Comm. Pure Appl. Math., v. 17, 1964, pp. 335-353. MR 29 \#4210.

$\dagger \dagger$ One of us [ELR] is indebted to Dr. Stanley Osher for a helpful discussion. 